\title{
Extracorporeal Membrane Oxygenation Causes Loss of Intestinal Epithelial Barrier in the Newborn Piglet
}

\author{
ASHISH R. KURUNDKAR, CHERYL R. KILLINGSWORTH, R. BRITT MCILWAIN, JOSEPH G. TIMPA, \\ YOLANDA E. HARTMAN, DONGNING HE, RAJENDRA K. KARNATAK, MARY L. NEEL, JOHN P. CLANCY, \\ G. M. ANANTHARAMAIAH, AND AKHIL MAHESHWARI
}

\begin{abstract}
Departments of Pediatrics [A.R.K., Y.E.H., D.H., R.K.K., M.L.N., J.P.C., A.M.], Medicine [C.R.K., G.M.A.], Pathology [A.M.], and Cell Biology [A.M.], University of Alabama at Birmingham (UAB), Birmingham, AL 35294; University Hospital Services [R.B.M., J.G.T.], University of Alabama at Birmingham, Birmingham, AL35294; Clinical Perfusion Education [R.B.M.], School of Allied Health Professions, University of Nebraska Medical Center, Omaha, NE 68198
\end{abstract}

\begin{abstract}
Extracorporeal membrane oxygenation (ECMO) is an important life-support system used in neonates and young children with intractable cardiorespiratory failure. In this study, we used our porcine neonatal model of venoarterial ECMO to investigate whether ECMO causes gut barrier dysfunction. We subjected 3-wk-old previously healthy piglets to venoarterial ECMO for up to $8 \mathrm{~h}$ and evaluated gut mucosal permeability, bacterial translocation, plasma levels of bacterial products, and ultrastructural changes in gut epithelium. We also measured plasma lipopolysaccharide (LPS) levels in a small cohort of human neonates receiving ECMO. In our porcine model, ECMO caused a rapid increase in gut mucosal permeability within the first $2 \mathrm{~h}$ of treatment, leading to a 6- to 10 -fold rise in circulating bacterial products. These changes in barrier function were associated with cytoskeletal condensation in epithelial cells, which was explained by phosphorylation of a myosin II regulatory light chain. In support of these findings, we also detected elevated plasma LPS levels in human neonates receiving ECMO, indicating a similar loss of gut barrier function in these infants. On the basis of these data, we conclude that ECMO is an independent cause of gut barrier dysfunction and bacterial translocation may be an important contributor to ECMO-related inflammation. (Pediatr Res 68: 128-133, 2010)
\end{abstract}

$\mathrm{E}$ xtracorporeal membrane oxygenation (ECMO) is an important cardiopulmonary life support system used in critically ill neonates and young children with respiratory failure, congenital heart disease, and overwhelming sepsis $(1,2)$. Although the availability of ECMO has reduced mortality in these patients by $\sim 80 \%$, concerns remain about the near-universal occurrence of a systemic inflammatory response syndrome (SIRS) during ECMO that is associated with considerable morbidity. ECMO-related SIRS is particularly severe in neonates (than in older children) $(3,4)$, manifesting with the first few hours of ECMO with hypotension, oliguria, decreased lung compliance, anasarca, and liver dysfunction.

Received January 21, 2010; accepted April 14, 2010.

Correspondence: Akhil Maheshwari, M.D., University of Alabama at Birmingham, 1670 University Boulevard, VH648C, Birmingham, AL 35294; e-mail: akhil@peds.uab.edu

Supported in part by the American Heart Association (AHA)/Suncoast Foundation, AHA grant 0665155B, American Gastroenterological Association 2006 Research Scholar Award, and NIH awards HD059142 and HD043397 [A.M.]. The work was made possible by Facilities Improvement Grant C06RR15490 from the National Center for Research Resources. There are no conflicts of interest to disclose.

A.R.K. and C.R.K. contributed equally to this study.
These changes frequently persist for several days and prolong the duration of ECMO (1). Understanding ECMO-related SIRS is a critical step in the development of effective antiinflammatory strategies.

Our understanding of the inflammatory effects of extracorporeal circulation is based primarily on studies on adult patients treated with cardiopulmonary bypass $(\mathrm{CPB})$ during cardiac surgery (5). However, direct extrapolation of data from CPB to ECMO is difficult because of major pathophysiological differences between the two modalities, such as the presence of defined ischemia-reperfusion sequences in CPB (related to the placement and release of aortic cross-clamps during cardiac surgery) but not in ECMO (5). To investigate the mechanisms of ECMO-related SIRS, we have developed a porcine neonatal model of ECMO where we subject previously healthy, 3-6 kg piglets to venoarterial ECMO in the laboratory (6). Porcine ECMO was associated with inflammatory changes similar to those reported in human studies, causing tachycardia, hypotension, and a generalized capillary leakiness manifesting as edema and ascites within the first few 2-4 $\mathrm{h}$ of initiation of the procedure. ECMO caused neutrophil activation, increased expression of inflammatory cytokines in plasma and tissues, and histopathological changes of inflammation (leukocyte infiltration) and microvascular injury (focal hemorrhages, edema) in diverse organs such as lung, intestine, liver, and kidney. We showed that the initiation of ECMO was associated with mast cell degranulation, which released large amounts of preformed proinflammatory cytokines such as tumor necrosis factor (TNF/TNF- $\alpha$ ) and mast cell-derived enzymes such as tryptase into the circulation. Because ECMO causes mast cell degranulation, which in turn, releases TNF, tryptase, and chymase that can disrupt the tight junctions of the intestinal epithelial cells (IECs) $(7,8)$, we postulate that ECMO induces gut barrier dysfunction which in turn leads to bacterial translocation and increased inflammation. In this study, we used our porcine model to test this hypothesis.

Abbreviations: CPB, cardiopulmonary bypass; ECMO, extracorporeal membrane oxygenation; IEC, intestinal epithelial cell; LPS, lipopolysaccharide; LTA, lipoteichoic acids; MLC, myosin light chain; SIRS, systemic inflammatory response syndrome 


\section{METHODS}

Neonatal porcine ECMO. The ECMO procedure has been described previously (6). Briefly, mixed-breed neonatal piglets of either gender weighing 3-6 $\mathrm{kg}$ were subjected to venoarterial ECMO after approval by the Institutional Animal Care and Use Committee. Sham animals received anesthesia, ventilation, cannulation, and heparinization similar to ECMO animals but were not connected to the E pump. Data in this study represent 8 animals each in sham and ECMO groups; 3 animals in each group were euthanized after $2 \mathrm{~h}$ of treatment and the remaining 5 after $8 \mathrm{~h}$. ECMO-related SIRS in these animals has been described previously (6).

The piglets received general anesthesia and mechanical ventilation (volume controlled, tidal volume $=15 \mathrm{~mL} / \mathrm{kg}, 10-15$ cycles $/ \mathrm{min}$, Hallowell $E M C$ 2000 ventilator) to maintain normal blood gases. Biomedicus $8 \mathrm{~F}$ cannulae (Medtronic, Minneapolis, MN) were inserted into the external jugular vein and the external carotid artery. Animals were heparinized to maintain activated clotting times (ACT) of 180-220 s. The ECMO system consisted of a Biomedicus BP-50 centrifugal pump (Medtronic, Shoreview, MN) and a Minimax hollow fiber oxygenator (Medtronic). Gas flow rates to membrane oxygenator were maintained at $0.5 \mathrm{~L} / \mathrm{min}$ of $100 \%$ oxygen, and flow rates in the circuit were advanced to $250 \mathrm{~mL} / \mathrm{min}$ or $1.5 \mathrm{~L} / \mathrm{min} / \mathrm{m}^{2}$.

Measurement of bacterial products in plasma and detection of bacteria in tissues. Lipopolysaccharide (LPS; Lonza, Walkersville, MD) and D-lactate (Bioassay Systems, Hayward, CA) were measured by commercially available colorimetric assays. Lipoteichoic acids (LTA) were detected in plasma by immunoprecipitation and western blotting using our previously described protocol (9). Aerobic/anaerobic blood cultures were processed at the hospital clinical laboratory. Bacteria were identified in intestinal tissue sections using the Brown and Brenn stain (10).

Measurement of gut mucosal permeability. We used an established protocol for ex vivo measurement of gut mucosal permeability (11). Briefly, freshly dissected jejunal or colonic mucosa was mounted in Ussing diffusion chambers $\left(0.5 \mathrm{~cm}^{2}\right.$; Physiologic Instruments, San Diego, CA $)$ in Krebs-Ringer bicarbonate buffer ( $\mathrm{pH} 7.4)$. We added FITC-dextran $4400(2.2 \mathrm{mg} / \mathrm{mL})$ to the apical reservoirs for $60 \mathrm{~min}$, after which media were collected from apical and basal chambers, and fluorescence was measured at $485 / 520 \mathrm{~nm}$. Tissue specimens were examined histopathologically to confirm epithelial integrity.

Real-time PCR. Expression of tight junction genes was measured by real-time PCR per our previously described protocol (12). Primers were designed using the Beacon Design software (Bio-Rad, Hercules, CA).

Transmission electron microscopy. Transmission electron microscopy (TEM) analyses were performed at the UAB High Resolution Imaging Facility. Tissue samples of jejunum $(\sim 10$ inches beyond the ligament of
Treitz) and the mid-transverse colon were harvested and fixed in $2 \%$ glutaraldehyde and then in $1 \%$ osmium tetroxide. Thin sections were examined with a JEOL 1200-EX/II transmission electron microscope at $80 \mathrm{kV}$. Computerassisted morphometric measurements of intercellular spaces were made using the Metamorph software (Molecular Devices, Sunnyvale, CA).

The permeability of IEC apical junctions was evaluated using the electrondense dye ruthenium red (13). Briefly, tissue samples were placed (luminal side up) in plastic chambers with an apical $1 \mathrm{~cm}^{2}$ circular opening and then exposed to $2.5 \%$ glutaraldehyde and $0.6 \%$ ruthenium red in $0.1 \mathrm{M}$ sodium cacodylate buffer, $\mathrm{pH} 7.3$ for $30 \mathrm{~min}$ and then to $2 \%$ osmium tetroxide and ruthenium red for $30 \mathrm{~min}$. Thin sections were evaluated by the presence of electron-dense RR deposits in the intercellular space between IECs. A total of 10 tight junctions were analyzed from each mucosal region.

Detection of myosin light chain $(M L C)$ and phospho-MLC. IECs were isolated by treating intestinal tissue with HBSS containing $0.2 \mathrm{M}$ ethylenediaminetetraacetic acid (EDTA), $10 \mathrm{mM}$ 2-mercaptoethanol, and phosphatase and kinase inhibitors (Sigma Chemical Co.) for $30 \mathrm{~min}$. Western blots were performed on cell lysates for phosphorylated (ser19) and total myosin II light chain (Cell Signaling Technology), using our previously described protocol $(12,14)$.

Plasma samples from human neonates treated with ECMO. The study was performed under appropriate oversight by the Institutional Review Board on discarded sera/plasma at the clinical laboratory from patients during the first $48 \mathrm{~h}$ of ECMO $(n=8)$. We included a control group $(n=8)$ with comparable GAs and birth weights who were admitted to the NICU for severe respiratory distress and received mechanical ventilation but did not require ECMO. Patients were not selected as controls if they had perinatal depression (5 min Apgar score $\leq 3$ ) or culture-positive early-onset infection. LPS concentrations were measured using the Limulus lysate assay as described earlier.

Statistical methods. Nonparametric tests were applied using the SigmaStat 3.1.1 software (Systat, Point Richmond, CA). The number of samples in each experiment is indicated in figure legends. Groups were compared by the Mann-Whitney $U$ (Wilcoxon rank-sum test) or the Kruskall-Wallis $H$ tests (with Dunn's posttest). Data are expressed as means \pm standard errors (SE). A $p$ value $<0.05$ was considered significant.

\section{RESULTS}

Bacterial translocation during ECMO. To determine whether ECMO caused important changes in gut barrier function, we first measured LPS and bacterial products in plasma
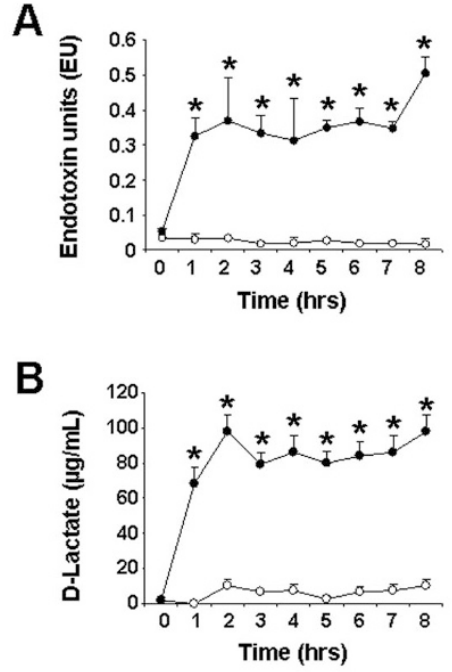
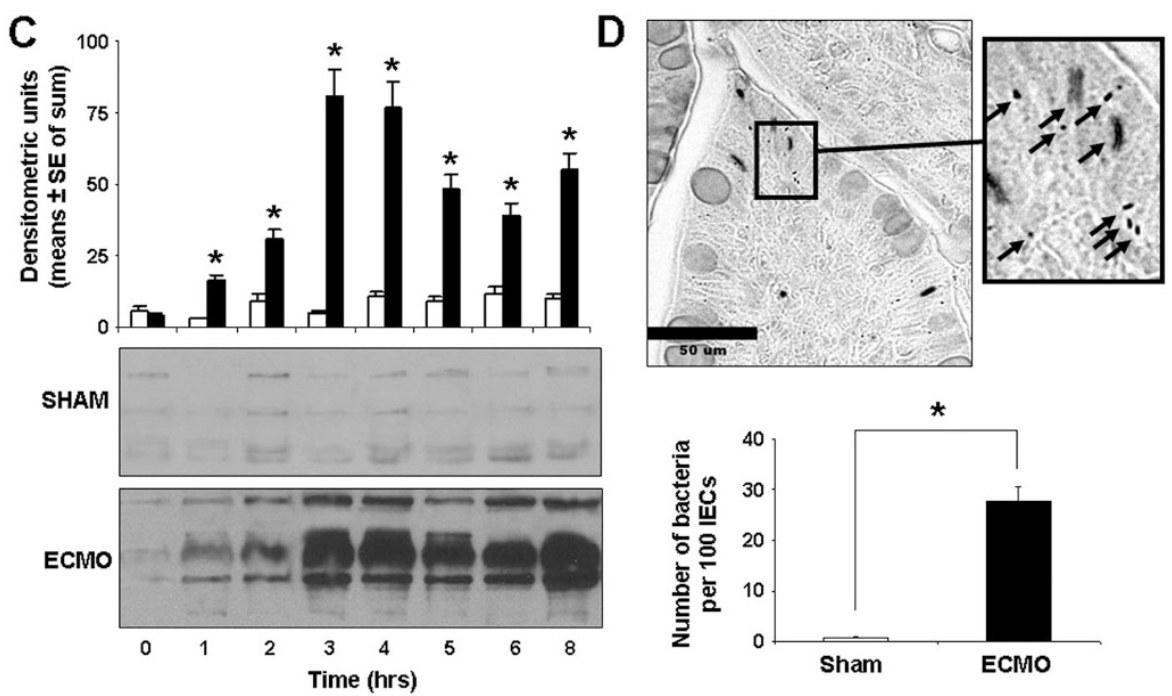

Figure 1. ECMO-mediated gut barrier dysfunction results in bacterial translocation. ( $A, B$ ) Plasma LPS (panel $A$ ) and D-lactate (panel $B$ ) levels increased rapidly during ECMO ( $n=5$ animals/group). Line diagrams show means \pm SEM values as a function of time. Black circles indicate data points from the ECMO group, whereas open circles depict the sham animals. * indicates $p<0.05$ when compared with sham at corresponding data points. $(C)$ Representative western blots show bacterial LTA in plasma. Bar graph of plasma lipoteichoic acid represents the sum of densities at each point (time after initiation of ECMO) for the 3 ECMO and 3 sham control animals. * indicates $p<0.05$ when compared with sham at corresponding data points. $(D)$ Brown and Brenn stain on intestinal sections (magnification $\times 400)$ reveals bacteria in the ECMO intestine. Inset $(\times 1500)$ shows rods and cocci (arrows). Bar diagram shows means \pm SE of the number of bacteria counted per 100 IECs in all 3 ECMO animals ( $2 \mathrm{~h}$ after initiation of ECMO) and sham controls. * indicates $p<0.001$ when compared with sham at corresponding data points. 
samples from sham and ECMO piglets. As shown in Figure $1 A$, plasma LPS increased rapidly within the first $2 \mathrm{~h}$ of ECMO but not in sham animals. Similarly, plasma concentrations of D-lactate, a specific product of prokaryotic metabolism, also increased within the first $2 \mathrm{~h}$ (Fig. 1B). We also probed for LTA, a component of the Gram-positive bacterial cell wall, in plasma samples by immunoprecipitation and western blotting and observed a similar increase in plasma LTA during ECMO (Fig. 1C). Blood cultures obtained from sham and ECMO animals remained negative, indicating that bacterial translocation remained limited at low levels during ECMO.

The rapid increase in circulating bacterial products we observed within 1-2 h of initiation of ECMO correlated with our earlier findings of a well-established inflammatory response within the first $2 \mathrm{~h}$ of ECMO (6). Therefore, to elucidate the mechanism of gut barrier dysfunction during $\mathrm{ECMO}$, we focused on the 2-h time point in subsequent experiments. To confirm that increased plasma levels of bacterial products were associated with increased bacterial translocation in the intestine, we stained intestinal tissue from sham and ECMO-treated animals for bacteria using the Brown and Brenn method. As shown in Fig. $1 D$, bacteria were readily detected ( $28 \pm 3$ bacteria/100 IECs) in the ECMO intestine, which was in sharp contrast with $<1$ bacterium seen per 100 IECs in the sham intestine $(p<0.05)$.

ECMO increases gut mucosal permeability. In vivo methods to assess gut mucosal permeability (15) were considered but not found suitable for our studies because of unpredictable effects of general anesthesia on bowel motility $(16,17)$. We used an ex vivo method to measure the penetration of FITCdextran, a marker of paracellular permeability, across freshly dissected and explanted specimens of jejunal and colonic mucosa (11). As seen in Figure 2, treatment with ECMO for $2 \mathrm{~h}$ increased permeability in the jejunal mucosa by $\sim 8$-fold $(1236 \pm 281$ in sham to $8226 \pm 1282$ fluorescence units in ECMO group; $p<0.05)$. Similarly, ECMO also increased permeability in the colonic samples $(6987 \pm 289$ units in ECMO versus $1364 \pm 436$ units in sham; $p<0.05$; effect not significantly different from jejunal samples).

Increased gut mucosal permeability during ECMO is not due to decreased expression of genes involved in the assembly of the tight junctions. To determine the mechanisms by which ECMO increased gut mucosal permeability, we first used a PCR array to measure the expression of 25 genes directly involved in tight junction assembly. As shown in Figure 3, the expression of occludin, several major claudins, and the zonula occludens protein-1 (ZO-1) remained unchanged or increased over sham after $2 \mathrm{~h}$ of ECMO. In view of these findings, we hypothesized that ECMO-induced changes in gut mucosal permeability were mediated via reorganization, rather than reduced expression, of junctional proteins, and therefore, we next evaluated ultrastructural changes by transmission electron microscopy.

ECMO is associated with ultrastructural abnormalities in the intestinal epithelium and increased junctional permeability. ECMO was associated with loss of intercellular contact with increased intercellular spaces in IECs, which con-
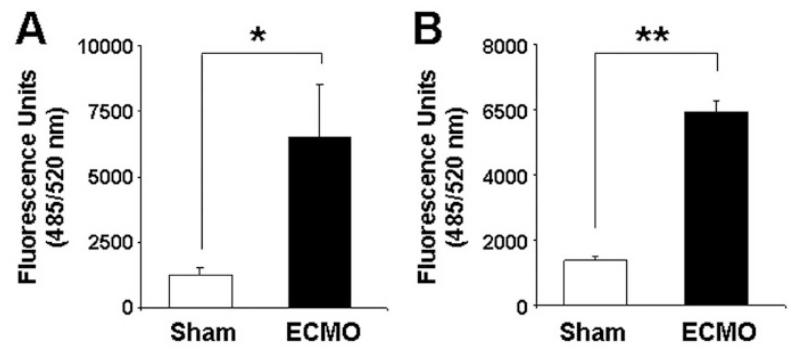

Figure 2. ECMO increases gut mucosal permeability. ECMO increased the mucosal permeability in explanted jejunal (panel A) and colonic (panel B) samples, measured as mucosal-to-basal penetration of FITC-dextran 4400 across tissue specimens in $60 \min (n=3-4$ tissue replicates per animal). $n=$ 3 animals per group; tissue replicates from each animal were averaged and used as a single data point. * indicates $p<0.01$, ** indicates $p<0.001$.

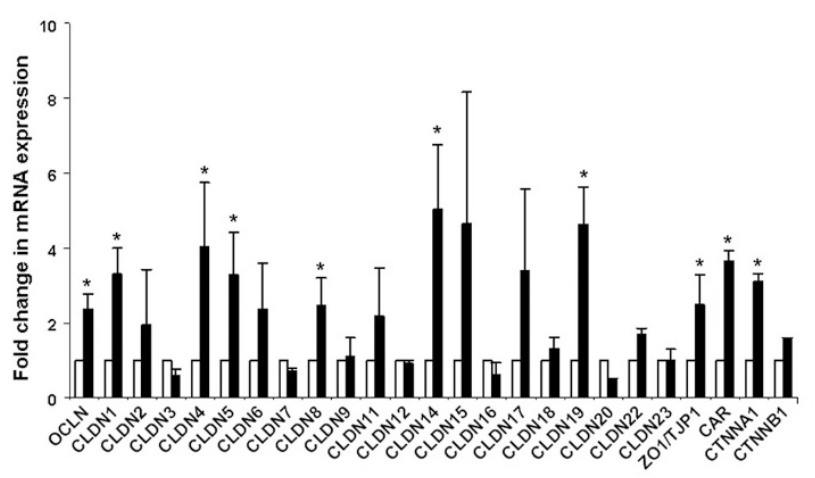

Figure 3. Increased gut mucosal permeability during ECMO is not due to decreased expression of genes involved in the assembly of the tight junctions. Bar diagram (means \pm SEM) depicts the expression of 25 porcine genes involved in tight junctions after $2 \mathrm{~h}$ of ECMO ( $\square)$, shown as fold change in mRNA expression above sham $(\square$, marked at fold change $=1) ; n=3$ animals/group. The expression of 20 of these 25 genes was increased (80\%). * indicates $p<0.05$ when compared with sham. Analysis for reduced gene expression by calculating 95\% confidence intervals did not show significant reductions. Claudin 10 mRNA was not detected in either sham or ECMO intestine. Abbreviations: OCLN, occludin; CLDN1-23, claudin 1-23; ZO1/TJP1, zonula occludens protein-1/tight junction protein-1; CAR, coxsackie-adenovirusreceptor homolog; CTNNA1, catenin (cadherin-associated protein), alpha 1; CTNNB1, catenin (cadherin-associated protein), beta 1 .

trasted with the close apposition of cell membranes in the sham intestine (sham $6.5 \pm 2 \mathrm{~nm}$ versus ECMO 101.2 \pm 11.7 nm; $p<0.05$; Fig. 4A). Similar changes were seen in the colon (not depicted).

To determine whether ECMO caused increased permeability of the apical junctional complex, we applied the electrondense dye ruthenium red to the apical surface of some explants before fixation for electron microscopy. As shown in Figure $4 B$, ruthenium red penetrated the apical junctions in the ECMO intestine and rendered those areas electron-dense (arrows), thereby indicating the functional disruption of these junctions.

ECMO causes cytoskeletal contraction in IECs. In the ECMO intestine, we noted electron-dense areas of perijunctional cytoskeletal condensation in the IECs (Fig. 5A). Consistent with previous reports (18), these changes were particularly prominent near tricellular junctions. 

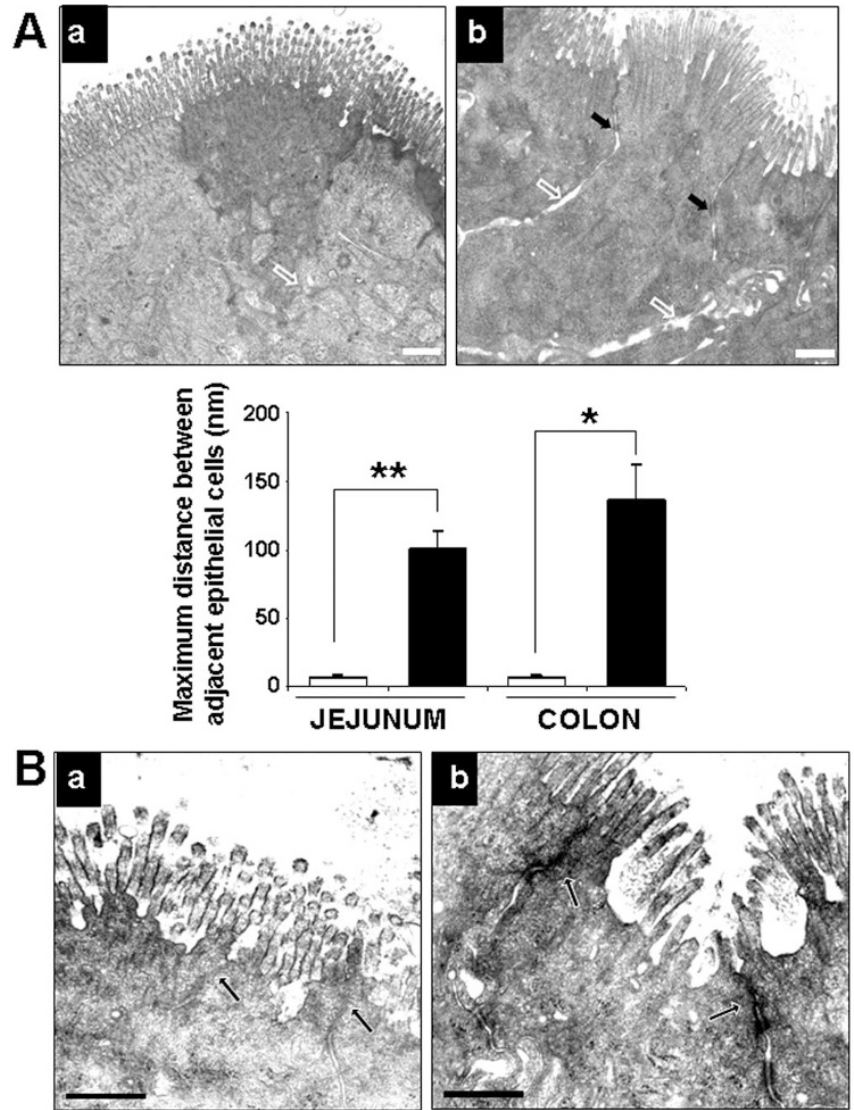

Figure 4. Ultrastructural changes in IECs in the ECMO intestine. (A) Loss of intercellular apposition in IECs in the ECMO intestine. Transmission electron micrographs (TEMs) of ( $a$ ) sham and (b) ECMO intestine showing increased separation of IECs after $2 \mathrm{~h}$ of ECMO (open arrows), except at desmosome sites (black arrows). Bar diagram (means $\pm \mathrm{SE}$ ) on the lower left summarizes the distances between adjacent epithelial cells in sham ( $\square$ ) and ECMO (ם) intestine. Distance bar represents $500 \mathrm{~nm}$. Data include junctions in two villi (or colonic crypts) in three randomly chosen areas. * indicates $p<$ 0.01 , ** indicates $p<0.001$. (B) Increased junctional permeability in the ECMO intestine: TEMs of $(a)$ sham and $(b)$ ECMO intestine show increased penetration of the electron-dense dye ruthenium red in the ECMO intestine (arrows). Distance bar represents $500 \mathrm{~nm}$.

To investigate the intracellular signaling events by which ECMO caused cytoskeletal contraction in IECs, we considered possible mechanisms involving MLC phosphorylation, rho-rho kinase-mediated signaling, and calcium/calmodulinactivated events $(7,19)$. Because inflammatory cytokines such as TNF can promote phosphorylation of the myosin II regulatory light chain (MLC) in IECs $(7,8,20)$, we considered MLC phosphorylation as the most plausible mechanism for the changes that we observed during ECMO-related SIRS. Using in silico sequence alignment, we first localized a conserved ser19 in porcine MLC that has been previously identified as a phosphorylation target in human and murine MLC. We next measured phosphorylated and total MLC in IECs by western blotting. As seen in Figure 5B, ECMO increased MLC phosphorylation in IECs.

Human neonatal ECMO is associated with increased plasma LPS concentrations. Finally, to confirm the clinical relevance of our findings in the porcine model, we measured plasma LPS levels in a small cohort of neonates during the

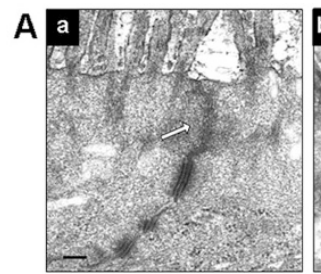

B

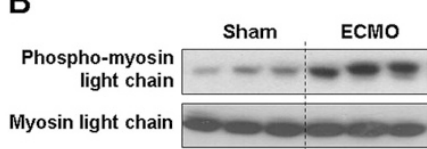

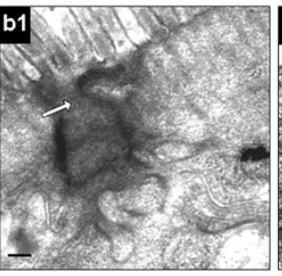
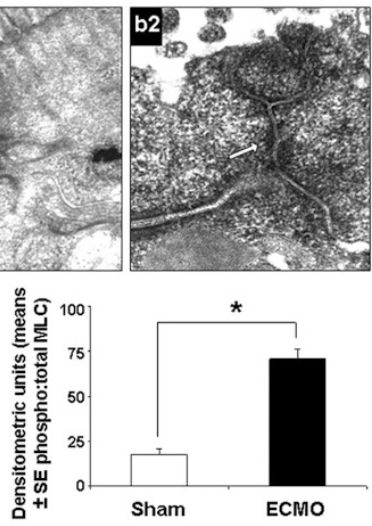

Figure 5. ECMO causes cytoskeletal contraction in IECs. (A) TEMs of (a) sham and $(b 1)$ ECMO intestine (magnification $\times 15,000$ ). Unlike the uniform appearance of the perijunctional IEC cytoskeleton in the sham intestine, IECs in the ECMO intestine show electron-dense areas of cytoskeletal condensation (arrows). High-magnification image $b 2$ shows prominent cytoskeletal condensation near a tricellular junction. $(B)$ ECMO increases the phosphorylation of myosin II light chain (MLC) in IECs. Western blots show increased phosphorylation of MLC in IECs. Bar diagrams show densitometric analysis (means $\pm \mathrm{SE}$ densitometric units) of the three bands (each representing a different animal) from sham and ECMO groups. Data were confirmed by repeating the blots. * indicates $p<0.01$, ** indicates $p<0.001$.

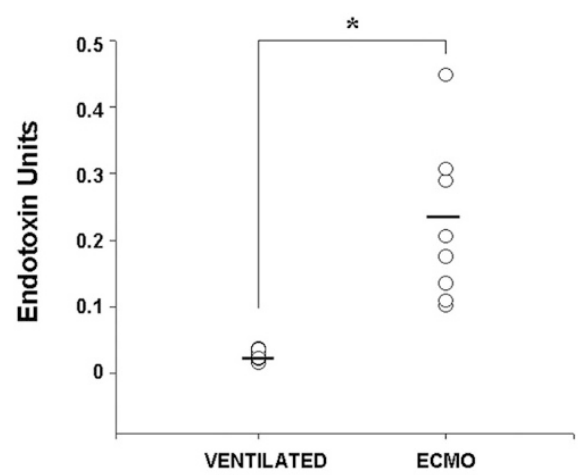

Figure 6. Human neonatal ECMO is associated with increased plasma LPS concentrations. Scatter plots showing higher plasma LPS concentrations in neonates during the first $48 \mathrm{~h}$ of ECMO therapy $(n=8)$ than in critically ill neonates on mechanical ventilation but not treated with ECMO $(n=8)$. * indicates $p<0.05$.

first day of ECMO therapy $(n=8)$. Diagnoses among the ECMO patients included meconium aspiration syndrome $(n=$ $7)$ and congenital diaphragmatic hernia $(n=2)$. Six neonates were on veno-venous ECMO and the other 3 on veno-arterial ECMO. The control group included neonates receiving mechanical ventilation for severe respiratory distress, who were not septic (blood culture was negative) and who did not require ECMO. The GA and birth weight of neonates on ECMO (GA, $39 \pm 2$ wk; birth weight, $3098 \pm 572 \mathrm{~g}$ ) were similar to the control group (GA, $38.6 \pm 2.7 \mathrm{wk}$; birth weight, $3289 \pm 342 \mathrm{~g}$ ). Plasma LPS concentrations were measured using the Limulus lysate assay. As seen in Figure 6, plasma levels were higher in neonates receiving ECMO therapy than those treated with mechanical ventilation alone. Although we cannot ascertain the contribution of the underlying disease to LPS elevations that we observed in these samples, these data provide indirect support for our findings in the porcine model. 


\section{DISCUSSION}

We present the first detailed investigation into the effects of ECMO on gut epithelial barrier and bacterial translocation. Existing evidence on ECMO-related SIRS emphasizes the role of neutrophils (activated in the ECMO circuit) (21-24), which cause diffuse microvascular injury and multiorgan dysfunction (21-25). In this study, we detected rapid deterioration of the intestinal barrier function and a consistent rise in circulating bacterial products almost immediately after the initiation of ECMO. Because bacterial products can independently initiate/ amplify all the major inflammatory pathways involved in ECMO-related SIRS (26), strategies to protect gut barrier function and thereby prevent bacterial translocation during ECMO merit careful evaluation. Our findings in the porcine model are consistent with previous observations of Piena et $a l$, who reported abnormalities in gut mucosal permeability in human neonates receiving ECMO (27). To assess mucosal integrity before initiating enteral feedings, these investigators measured intestinal permeability by measuring urinary excretion of enterally administered lactulose, rhamnose, D-xylose, and 3-O-methyl-D-glucose and noted abnormal mucosal permeability in 13 of their 16 patients. Similarly, Hirthler et al. (28) have previously reported increased plasma LPS levels in neonates who showed signs of inflammation $\geq 36 \mathrm{~h}$ after the initiation of ECMO. Bacterial translocation has also been noted following CPB used for cardiac surgery, where it correlated with ischemia-reperfusion injury sustained during the placement and subsequent release of aortic crossclamps $(5,29)$.

In this study, we have identified cytoskeletal condensation in IECs as a mechanism for the rapid disruption of the gut epithelial barrier during ECMO. Phosphorylation of the MLC and consequent cytoskeletal contraction are emerging as a common, central event in the disruption of the epithelial barrier in diverse conditions such as inflammatory bowel disease, graft versus host disease, and infectious enterocolitis $(7,19)$. In epithelial cells, tight junctions are affiliated with an apical perijunctional actin belt, which may contract to generate mechanical forces strong enough to retract the plasma membrane and cause internalization of various components of the apical junctional complex into subapical cytosolic compartments $(30,31)$. Although inflammatory mediators such as TNF are the likely cause of MLC phosphorylation and cytoskeletal contraction in IECs during ECMO, additional procedure-related factors such as exposure to citrate in the anticoagulant solutions in banked blood also need careful evaluation. Citrate can deplete extracellular calcium (32), which, in turn, can trigger cytoskeletal condensation and disassembly of tight junctions (33-35). Anticoagulant solutions assume great importance in neonates and young infants receiving ECMO because volumes of the ECMO circuit are often 200-300\% larger than a neonate's circulating blood volume, resulting in massive exposures to these reagents per unit weight $(3,4)$. Although most anticoagulants can be eliminated to a large extent by saline prewashing of the red blood cells $(\mathrm{RBC})$, the procedure may increase RBC osmotic fragility and hemolysis in centrifugal pumps (36). Rigorous clinical studies are needed to compare the effects of various anticoagulant solutions on ECMO-related SIRS.

This study provides us with two important conclusions that might have been difficult to draw in clinical studies: (1) by subjecting previously healthy piglets to ECMO, we established ECMO as an independent cause of gut barrier dysfunction. In clinical studies, the relative contribution of ECMO to barrier dysfunction cannot be dissected from that of the underlying disease that led to ECMO in the first place; bacterial translocation is common during hypoxemic respiratory failure, congestive cardiac failure, intractable sepsis, and shock, conditions that form the commonest indications for $\operatorname{ECMO}(1,26)$; (2) development of gut barrier dysfunction and bacterial translocation early during the course of ECMO suggests that barrier dysfunction is more likely to be a primary initiator/ amplifier of ECMO-related SIRS and not merely an epiphenomenon reflecting loss of mucosal integrity as a part of ECMO-related multisystem dysfunction. Unlike most intestinal/systemic conditions associated with gut barrier dysfunction, ECMO is uniquely placed for intervention because a finite temporal window is frequently available to the clinician to initiate preventive measures even as the procedure is being started.

ECMO-related SIRS cannot be prevented merely by improvements in the ECMO circuit. The physical characteristics of the ECMO circuit such as fluid dynamics, blood volume to surface area ratio, and the material's affinity for fibrinogen determine its propensity for contact activation of inflammatory pathways (37). Although smoother circuits can be designed, the structure of the membrane oxygenator calls for conflicting profiles - the necessity for the gas exchange obligates thin blood films and turbulence, factors that also favor contact activation. Thus, even though improved silicone oxygenators have shown modest benefit in reducing platelet and contact activation, these improvements face a mathematical bottleneck where reduction in turbulence and blood surface interaction compete with the gas exchange capacity of the oxygenator (38). In this context, elucidation of the inflammatory pathways involved in ECMO-related SIRS is a critical step in the development of effective anti-inflammatory therapies. As potent inhibitors of MLC kinase are now available (39), the identification of MLC phosphorylation as a likely mechanism of gut barrier dysfunction during ECMO indicates a need for further investigation of this pathway in preclinical and clinical settings.

\section{REFERENCES}

1. Hansell DR 2003 Extracorporeal membrane oxygenation for perinatal and pediatric patients. Respir Care 48:352-362; discussion 363-356

2. Coppola CP, Tyree M, Larry K, DiGeronimo R 2008 A 22-year experience in globa transport extracorporeal membrane oxygenation. J Pediatr Surg 43:46-52

3. Butler J, Pathi VL, Paton RD, Logan RW, MacArthur KJ, Jamieson MP, Pollock JC 1996 Acute-phase responses to cardiopulmonary bypass in children weighing less than 10 kilograms. Ann Thorac Surg 62:538-542

4. Kozik DJ, Tweddell JS 2006 Characterizing the inflammatory response to cardiopulmonary bypass in children. Ann Thorac Surg 81:S2347-S2354

5. Brix-Christensen V 2001 The systemic inflammatory response after cardiac surgery with cardiopulmonary bypass in children. Acta Anaesthesiol Scand 45:671-679

6. McILwain RB, Timpa JG, Kurundkar AR, Holt DW, Kelly DR, Hartman YE, Neel ML, Karnatak RK, Schelonka RL, Anantharamaiah GM, Killingsworth CR, Maheshwari A 2010 Plasma concentrations of inflammatory cytokines rise rapidly 
during ECMO-related SIRS due to the release of pre-formed stores in the intestine. Lab Invest 90:128-139

7. Clayburgh DR, Barrett TA, Tang Y, Meddings JB, Van Eldik LJ, Watterson DM, Clarke LL, Mrsny RJ, Turner JR 2005 Epithelial myosin light chain kinasedependent barrier dysfunction mediates $\mathrm{T}$ cell activation-induced diarrhea in vivo. J Clin Invest 115:2702-2715

8. Brown GR, Lindberg G, Meddings J, Silva M, Beutler B, Thiele D 1999 Tumor necrosis factor inhibitor ameliorates murine intestinal graft-versus-host disease. Gastroenterology 116:593-601

9. Maheshwari A, Voitenok NN, Akalovich S, Shaik SS, Randolph DA, Sims B, Patel RP, Killingsworth CR, Fallon MB, Ohls RK 2009 Developmental changes in circulating IL-8/CXCL8 isoforms in neonates. Cytokine 46:12-16

10. Brown JH, Brenn L 1931 A method for the differential staining of Gram-positive and Gram-negative bacteria in tissue sections. Bull Johns Hopkins Hosp 48:69-73

11. Wang Q, Hasselgren PO 2002 Heat shock response reduces intestinal permeability in septic mice: potential role of interleukin-10. Am J Physiol Regul Integr Comp Physiol 282:R669-R676

12. Shaik SS, Soltau TD, Chaturvedi G, Totapally B, Hagood JS, Andrews WW, Athar M, Voitenok NN, Killingsworth C, Patel RP, Fallon MB, Maheshwari A 2009 Low-intensity shear stress increases endothelial ELR + CXC chemokine production via a FAK-P38beta MAPK-NF-kappa B pathway. J Biol Chem 284:5945-5955

13. Soler AP, Miller RD, Laughlin KV, Carp NZ, Klurfeld DM, Mullin JM 1999 Increased tight junctional permeability is associated with the development of colon cancer. Carcinogenesis 20:1425-1431

14. Maheshwari A, Kurundkar AR, Shaik SS, Kelly DR, Hartman Y, Zhang W, Dimmitt R, Saeed S, Randolph DA, Aprahamian C, Datta G, Ohls RK 2009 Epithelial cells in fetal intestine produce chemerin to recruit macrophages. Am J Physiol Gastrointest Liver Physiol 297:G1-G10

15. Shulman RJ, Eakin MN, Czyzewski DI, Jarrett M, Ou CN 2008 Increased gastrointestinal permeability and gut inflammation in children with functional abdominal pain and irritable bowel syndrome. J Pediatr 153:646-650

16. Anderson DL, Bartholomeusz FD, Kirkwood ID, Chatterton BE, Summersides G, Penglis S, Kuchel T, Sansom L 2002 Liquid gastric emptying in the pig: effect of concentration of inhaled isoflurane. J Nucl Med 43:968-971

17. Hietbrink F, Besselink MG, Renooij W, Leenen LP 2007 Pitfalls in gastrointestinal permeability measurement in ICU patients. Intensive Care Med 33:2216

18. Madara JL, Barenberg D, Carlson S 1986 Effects of cytochalasin D on occluding junctions of intestinal absorptive cells: further evidence that the cytoskeleton may influence paracellular permeability and junctional charge selectivity. J Cell Biol 102:2125-2136

19. Turner JR 2006 Molecular basis of epithelial barrier regulation: from basic mechanisms to clinical application. Am J Pathol 169:1901-1909

20. Zolotarevsky Y, Hecht G, Koutsouris A, Gonzalez DE, Quan C, Tom J, Mrsny RJ, Turner JR 2002 A membrane-permeant peptide that inhibits MLC kinase restores barrier function in in vitro models of intestinal disease. Gastroenterology 123:163-172

21. Fortenberry JD, Bhardwaj V, Niemer P, Cornish JD, Wright JA, Bland L 1996 Neutrophil and cytokine activation with neonatal extracorporeal membrane oxygenation. J Pediatr 128:670-678

22. Underwood MJ, Pearson JA, Waggoner J, Lunec J, Firmin RK, Elliot MJ 1995 Changes in "inflammatory" mediators and total body water during extra- corporeal membrane oxygenation (ECMO). A preliminary study. Int J Artif Organs 18:627-632

23. Graulich J, Sonntag J, Marcinkowski M, Bauer K, Kossel H, Buhrer C, Obladen M, Versmold HT 2002 Complement activation by in vivo neonatal and in vitro extracorporeal membrane oxygenation. Mediators Inflamm 11:69-73

24. Graves ED 3rd, Loe WA, Redmond CR, Falterman KW, Arensman RM 1989 Extracorporeal membrane oxygenation as treatment of severe meconium aspiration syndrome. South Med J 82:696-698

25. DePuydt LE, Schuit KE, Smith SD 1993 Effect of extracorporeal membrane oxygenation on neutrophil function in neonates. Crit Care Med 21:1324-1327

26. Gatt M, Reddy BS, MacFie J 2007 Review article: bacterial translocation in the critically ill-evidence and methods of prevention. Aliment Pharmacol Ther 25:741-757

27. Piena M, Albers MJ, Van Haard PM, Gischler S, Tibboel D 1998 Introduction of enteral feeding in neonates on extracorporeal membrane oxygenation after evaluation of intestinal permeability changes. J Pediatr Surg 33:30-34

28. Hirthler M, Simoni J, Dickson M 1992 Elevated levels of endotoxin, oxygen-derived free radicals, and cytokines during extracorporeal membrane oxygenation. J Pediatr Surg 27:1199-1202

29. Walker LK, Short BL, Traystman RJ 1996 Impairment of cerebral autoregulation during venovenous extracorporeal membrane oxygenation in the newborn lamb. Crit Care Med 24:2001-2006

30. Ivanov AI, McCall IC, Parkos CA, Nusrat A 2004 Role for actin filament turnover and a myosin II motor in cytoskeleton-driven disassembly of the epithelial apical junctional complex. Mol Biol Cell 15:2639-2651

31. Shen L, Black ED, Witkowski ED, Lencer WI, Guerriero V, Schneeberger EE, Turner JR 2006 Myosin light chain phosphorylation regulates barrier function by remodeling tight junction structure. J Cell Sci 119:2095-2106

32. Kaul TK, Bhatnagar NK, Mercer JL 1989 Plasma albumin and calcium levels following cardiopulmonary bypass. Int J Artif Organs 12:461-465

33. Cereijido M, Robbins ES, Dolan WJ, Rotunno CA, Sabatini DD 1978 Polarized monolayer formed by epithelial cells on a permeable and translucent support. J Cell Biol 77:853-880

34. Siliciano JD, Goodenough DA 1988 Localization of the tight junction protein, ZO-1, is modulated by extracellular calcium and cell-cell contact in Madin-Darby canine kidney epithelial cells. J Cell Biol 107:2389-2399

35. Ivanov AI, Nusrat A, Parkos CA 2004 Endocytosis of epithelial apical junctiona proteins by a clathrin-mediated pathway into a unique storage compartment. Mol Biol Cell 15:176-188

36. Masalunga C, Cruz M, Porter B, Roseff S, Chui B, Mainali E 2007 Increased hemolysis from saline pre-washing RBCs or centrifugal pumps in neonatal ECMO. J Perinatol 27:380-384

37. Hennessy VL Jr, Hicks RE, Niewiarowski S, Edmunds LH Jr, Colman RW 1977 Function of human platelets during extracorporeal circulation. Am J Physiol 232:H622-H628

38. Baier RE 1977 The organization of blood components near interfaces. Ann N Y Acad Sci 283:17-36

39. Feighery LM, Cochrane SW, Quinn T, Baird AW, O'Toole D, Owens SE, O’Donoghue D, Mrsny RJ, Brayden DJ 2008 Myosin light chain kinase inhibition: correction of increased intestinal epithelial permeability in vitro. Pharm Res 25:1377-1386 